Z Gerontol Geriat 2019·52:111-112 https://doi.org/10.1007/s00391-019-01515-5 Online publiziert: 8. Februar 2019

(c) Springer Medizin Verlag $\mathrm{GmbH}$, ein Teil von Springer Nature 2019

CrossMark

\author{
Jürgen M. Bauer ${ }^{1,5}$. Michael Denkinger ${ }^{2,6} \cdot$ Sebastian Voigt-Radloff ${ }^{3}$. \\ Hans Jürgen Heppner ${ }^{4,7}$ \\ ${ }^{1}$ Geriatrisches Zentrum, Universität Heidelberg, Heidelberg, Deutschland \\ ${ }^{2}$ Geriatrie, Geriatrisches Zentrum Ulm/Alb-Donau, Universität Ulm, Ulm, Deutschland \\ ${ }^{3}$ Institut für Evidenz in der Medizin (für Cochrane Deutschland Stiftung), Medizinische Fakultät, Zentrum \\ für Geriatrie und Gerontologie Freiburg, Universitätsklinikum Freiburg, Freiburg, Deutschland \\ ${ }^{4}$ Klinik für Geriatrie, HELIOS Klinikum Schwelm, Schwelm, Deutschland \\ ${ }^{5}$ Agaplesion Bethanien Krankenhaus Heidelberg, Heidelberg, Deutschland \\ ${ }^{6}$ Agaplesion Bethesda Klinik Ulm, Ulm, Deutschland \\ ${ }^{7}$ Lehrstuhl Geriatrie, Universität Witten/Herdecke, Witten, Deutschland
}

\title{
Aktuelle Cochrane-Reviews, kommentiert aus geriatrischer Perspektive
}

\section{Orientierung in Zeiten der Informationsoverkills}

Das Internet erlaubt es uns, auf eine Vielzahl medizinischer Datenbanken zuzugreifen und in wenigen Sekunden Originaltexte zu erhalten - falls wir im Besitz der entsprechenden Berechtigungen sind. Der Mehrzahl der in Praxis und Klinik tätigen Kollegen verfügt jedoch nicht über Onlinezugänge, die es ermöglichen, weltweit in wissenschaftlichen Zeitschriften publizierte Artikel herunterzuladen. Die Kosten für den Download einzelner Artikel sind erheblich und der Zugriff auf ganze Zeitschriftenpakete unerschwinglich. Die Zahl von Open-Access-Journalen wächst zwar rasch, ihr Inhalt kann in der überwiegenden Mehrzahl gegenwärtig jedoch noch nicht mit den etablierten Zeitschriften mithalten. Zusätzlich wurde das Feld der Open-AccessJournale aufgrund der Praktiken einer Gruppe von Zeitschriften, die man als „predatory journals“ bezeichnet, partiell diskreditiert. Unter Letzteren versteht man als betrügerisch angesehene Zeitschriften, die die üblichen Open-AccessGebühren erheben und vorgeben, wissenschaftlich vollwertig zu arbeiten, jedoch nicht die in diesem Kontext erforderlichen redaktionellen und publizistischen Leistungen wie einen seriösen „peer review“ erbringen. Auf diese Wei- se untergraben sie die Glaubwürdigkeit der Wissenschaft und liefern Argumente, die den Wert und Einfluss anspruchsvoller Forschung und Lehre zu verringern beabsichtigen.

Wie diese Beispiele veranschaulichen, ist der Zugriff auf verlässliche aktuelle Informationen, welche die Entscheidungsfindung hinsichtlich der im Einzelfall indizierten Diagnostik und Therapie erleichtern, trotz des weiterhin rasch wachsenden Informationsangebots des Internets schwierig. Leitlinien bieten hier zwar eine wertvolle Orientierung, jedoch führt der aufwendige Erstellungsprozess dazu, dass die Zeiträume zwischen dem Erscheinen der eingeschlossenen wissenschaftlichen Literatur und der Veröffentlichung der Leitlinie erheblich sein können. Ferner dauert es in der Regel mehrere Jahre, bis die erforderliche Überarbeitung erscheint. Hier können sich Diskrepanzen zwischen der Empfehlung einer Leitlinie und dem aktuellen Stand der Wissenschaft ergeben. Die Kollegin resp. der Kollege, die/der nicht regelmäßig die wissenschaftlichen Neuveröffentlichungen auf diesem Gebiet verfolgt, ist diesem Sachverhalt ausgeliefert.

Mit unserer neuen Rubrik „Cochrane Corner Geriatrie“ möchten wir hel- fen, diese Wissenslücke $\mathrm{zu}$ schließen Das Format der Cochrane Corner ist international für mehrere medizinische Disziplinen etabliert, beinhaltet die Übersetzung und Kommentierung aktueller Cochrane-Evidenz für das jeweilige Fach und zielt auf die Verbesserung des Evidenz-Praxis-Transfers ab. Jeder Beitrag der neuen Kategorie Cochrane Corner Geriatrie umfasst zum einen die Abstract-Übersetzung eines aktuellen und für die Geriatrie relevanten Cochrane-Reviews sowie seine anschließende Einordnung für die geriatrische Versorgung. Dabei sollen die für die Geriatrie oftmals zu vereinfachenden Schlussfolgerungen in den Kontext der komplexen Versorgung multimorbider und funktionell mehrfach eingeschränkter älterer Patienten gestellt werden. Diesem neuartigen Projekt liegt eine Kooperation mit dem Deutschen Cochrane Zentrum in Freiburg zugrunde, die sich im Rahmen des vom Land Baden-Württemberg geförderten wissenschaftlichen Projektes "Medikation und Lebenssituation im Alter" entwickelt hat, und an dem die Universitäten Heidelberg, Freiburg und Ulm beteiligt sind.

Im Rahmen dieses Projektes konnten wir auch, beispielhaft für zwei Cochrane- 
Reviews, eine kooperative Strategie zur stetigen Aktualisierung vereinbaren. Die Recherchespezialisten von Cochrane International liefern zum jeweiligen Review alle drei Monate die aktuellen Primärstudientreffer. Projektmitarbeiter sichten die Abstracts dieser Studien und analysieren relevante Volltexte daraufhin, ob sie die Schlussfolgerung des Reviews verändern würden. Falls dies der Fall ist, werden diese Studien in einen Beitrag für die Zeitschrift für Gerontologie und Geriatrie eingearbeitet.

Da in den allermeisten Dokumenten altersbezogene Empfehlungen gegenwärtig noch die Ausnahme darstellen, fokussieren die Kommentierungen auf die Besonderheiten des geriatrischen Patienten. Allen Projektbeteiligten ist sehr bewusst, dass Therapieentscheidungen nicht ausschließlich auf Leitlinien und CochraneReviews basieren können, sondern im Sinne der patientenorientierten und evidenzbasierten Geriatrie immer die besonderen Umstände des einzelnen Patienten reflektieren müssen. Unsere Beiträge im Rahmen der Cochrane Corner können hier hoffentlich wertvolle Anregungen für die therapeutische Entscheidungsfindung geben.

\section{Korrespondenzadresse}

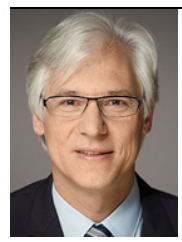

Prof. Dr. med. Jürgen M. Bauer Agaplesion Bethanien Krankenhaus Heidelberg Rohrbacher Str. 149, 69126 Heidelberg, Deutschland juergen.bauer@bethanienheidelberg.de

Interessenkonflikt. J.M. Bauer, M. Denkinger, S. Voigt-Radloff und H.J. Heppner geben an, dass kein Interessenkonflikt besteht.

\section{Preis zur Förderung der interdisziplinären Altersforschung}

Die Deutsche Gesellschaft für Geriatrie (DGG) e.V. schreibt diesen Preis für das Jahr 2019 aus. Der Preis wird gestiftet aus dem Vermögen des früheren Dachverbandes der Gerontologischen und Geriatrischen Wissenschaftlichen Gesellschaften Deutschlands e. V. (DVGG).

Gefördert werden sollen Projekte, die in Form von Vorträgen oder Posterpublikationen auf den Jahreskongressen der Deutschen Gesellschaft für Geriatrie vorgetragen werden, die in besonderer Weise einen interdisziplinären oder translationalen Ansatz zeigen.

\section{Das Preisgeld beträgt z. Z. 2.000€}

Mit einem regulären Beitrag für den jeweiligen Jahreskongress DGG können die Einreichenden als Einzelpersonen oder als Arbeitsgruppe/Autorengruppe angeben, ob sie sich um den Preis bewerben wollen.

An der endgültigen Preisvergabe nehmen nur als Poster oder Vortrag angenommene Beiträge teil. Über die Annahme als Vortrag oder Poster entscheidet das Auswahlkomitee des Geriatriekongresses. Orientiert an der Deadline des Kongresses, jedoch spätestens 6 Wochen vor Kongressbeginn, ist neben dem Abstract eine Kurzübersicht des Beitrages in elektronischer Form einzureichen. Die Kurzübersicht soll wie eine herkömmliche Publikation gegliedert sein und sollte 700 Worte (zzgl. Abstract), 5 Abbildungen bzw. Tabellen sowie 5 Literaturstellen nicht überschreiten. Alternativ, aber nicht zwingend notwendig kann auch ein Publikationsmanuskript des Beitrages eingereicht werden.

Die Deutsche Gesellschaft für Geriatrie gibt ihre Vorauswahl zur Entscheidung an das Preiskomitee des Preises zur Förderung der interdisziplinären Altersforschung weiter.

Das Preiskomitee besteht aus Prof. Dr. Dr. Kolb (Koordination und Vorsitz). Prof. Dr. Heppner (Amtierender Präsident der DGG und Vertreter Geriatrie), Prof. Dr. Wöstmann (Vertreter der Zahnmedizin), Prof. Dr. Oswald (Vertreter Gerontologie).

Die Preisverleihung wird anlässlich der 31. Jahrestagung der Deutschen Gesellschaft für Geriatrie e. V. vom 05.09. bis 07.09.2019 in Frankfurt/Main stattfinden.

Weitere Auskünfte erteilt auf Wunsch:

Prof. Dr. Dr. Kolb

Vorsitzender des Preiskomitees

Bonifatius Hospital Lingen Fachbereich Geriatrie Wilhelmstraße 13

49808 Lingen/Ems

E-Mail: gerald.kolb@hospital-lingen.de 